\title{
Mining 2:2 Complexes from 1:1 Stoichiometry: Formation of Cucurbit[8]uril-Diarylviologen Quaternary Complexes Favored by Electron-Donating Substituents
}

\author{
Guanglu Wu, ${ }^{\dagger, \S}$ Magdalena Olesińska, ${ }^{\dagger, \S}$ Yuchao Wu, ${ }^{\dagger}$ Dijana Matak-Vinkovic, ${ }^{\ddagger}$ \\ and Oren A. Scherman*, ${ }^{*}+0$
}
${ }^{\dagger}$ Melville Laboratory for Polymer Synthesis, Department of Chemistry, University of Cambridge, Lensfield Road, Cambridge CB2 1EW, U.K.

${ }^{\ddagger}$ Department of Chemistry, University of Cambridge, Lensfield Road, Cambridge CB2 1EW, U.K.

\section{Supporting Information}

\begin{abstract}
A 1:1 binding stoichiometry of a host-guest complex need not consist of a single host and guest. Diarylviologens containing electron-donating substituents complexed with cucurbit[8] uril $(\mathrm{CB}[8])$ in a 1:1 stoichiometry exhibit abnormally large binding enthalpies compared to typical enthalpy changes observed for 1:1 binary complexes. Here, several $\mathrm{CB}[8]$-mediated host-guest complexes, which were previously reported as 1:1 binary complexes, are verified to be $2: 2$ quaternary complexes by a combination of isothermal titration calorimetry, ${ }^{1} \mathrm{H}$, NOESY, and ROESY NMR, and ion mobility mass spectrometry, clearly indicating a binding motif of two partially overlapping diarylviologens held in place with two $\mathrm{CB}[8]$ molecules. Formation of $2: 2$ quaternary complexes is favored by electron-donating substituents, while electron-withdrawing substituents typically result in

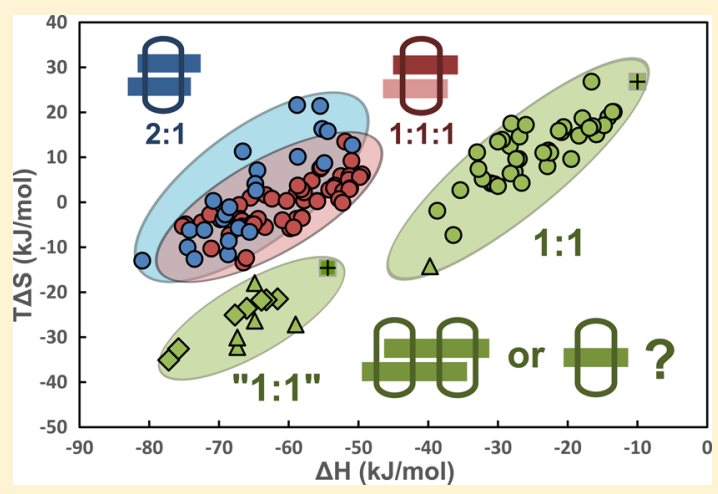
1:1 binary complexes. The stacking of two highly conjugated diarylviologens in one quaternary motif affords the complexes enhanced conductance when considered as a single-molecular conductor. Moreover, an additional conducting signal previously observed for this "supramolecular" conductor can be readily understood with our 2:2 complexation model, corresponding to a parallel conductance pathway. Therefore, a 2:2 quaternary complex model grants a greater understanding of such supramolecular complexes, enabling the design of engineered, hierarchical
\end{abstract} structures and functional materials.

\section{INTRODUCTION}

Cucurbit $[n]$ urils $(\mathrm{CB}[n])$ are a family of synthetic macrocyclic host molecules capable of encapsulating a wide range of organic guests in aqueous solution with exceptional affinity. ${ }^{1-3}$ Among cucurbituril homologues, $\mathrm{CB}[8]$ is versatile on account of its ability to form ternary complexes by accommodating two different guests (e.g., methyl viologen and naphthol ${ }^{4,5}$ ) or two identical guests (e.g., phenylalanine ${ }^{6}$ ). Exploration of new binding motifs is of great importance, as discovery of $\mathrm{CB}[8]$ mediated ternary complexes has led to unique applications in molecular ${ }^{7}$ or chiral $^{8}$ recognition, supramolecular catalysis, ${ }^{9}$ and engineered nanostructures. ${ }^{10,11}$ While detection of binding stoichiometry is useful in the investigation of new supramolecular complexes, it does not always reflect the exact binding motif at a molecular level. For instance, a host-guest binding stoichiometry of $1: 1$ only suggests that the complex contains equal amounts of both host and guest, which in practice can be 1:1, 2:2, or even a combination of $n: n(n=1,2$, $3, \ldots)$ in the case of a supramolecular polymer. Therefore, other binding features should be exploited in order to uncover the exact binding at a molecular level.
In aqueous solution, the host-guest binding of cucurbituril is mainly driven by the release of energetically frustrated cavitybound water ("high-energy water"), ${ }^{12-14}$ whereby free energy is contributed mostly from enthalpy and is directly related to the amount of water molecules being displaced. For example, the formation of a binary complex in aqueous solution typically releases less heat than the formation of a ternary complex, as only one guest displaces a smaller number of cavity-bound water molecules. Thus, the enthalpy change from binding can provide insight into the exact binding motif of $\mathrm{CB}[8]$-mediated host-guest complexation.

We assembled previously published thermodynamic binding data (Tables $\mathrm{S} 1-\mathrm{S} 3$ ) relating to $\mathrm{CB}[8]$-mediated host-guest complexation, ${ }^{5,13,16-27}$ all of which were measured by isothermal titration calorimetry (ITC) in buffered solution. Typical ITC data readily supply complexation information including binding stoichiometry, enthalpy changes $(\Delta H)$, and binding constants $\left(K_{\mathrm{a}}\right)$, from which we can further deduce

Received: December 20, 2016

Published: February 15, 2017 
changes in both the Gibbs free energy $(\Delta G)$ and entropy $(\Delta S)$. Thermodynamic data plotted in Figure 1 were obtained by

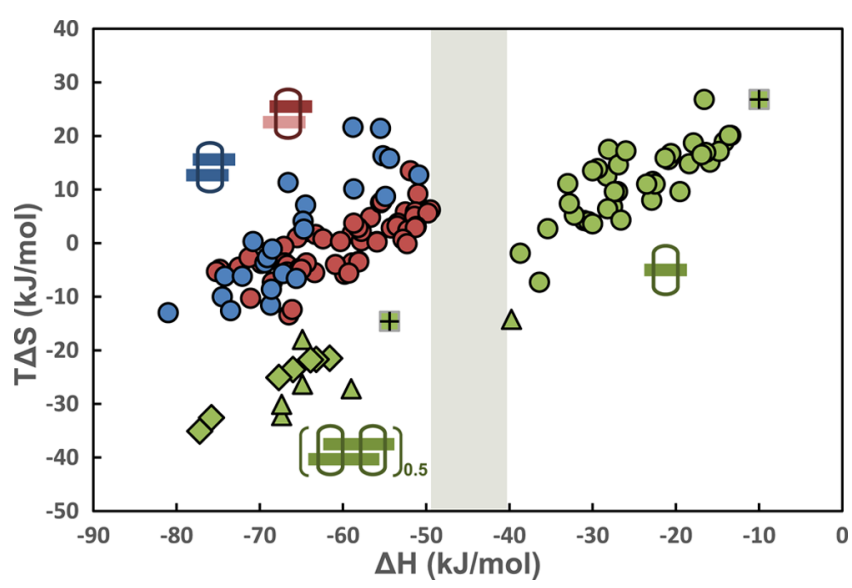

Figure 1. Thermodynamic data for $\mathrm{CB}[8]$-mediated host-guest complexation determined by ITC in buffered aqueous solution, including 2:1 homoternary complexes (blue), 1:1:1 heteroternary complexes (red), and complexes with 1:1 binding stoichiometry (green). Included in the 1:1 stoichiometry data are complexes exhibiting abnormally large enthalpy changes consisting of diarylviologens ${ }^{15}$ (diamonds), sequence-specific peptides ${ }^{16}$ (triangles), and an azobenzene derivative ${ }^{17}$ (squared-crosses). N.B. Data plotted for 1:1:1 heteroternary complexes are the sum of enthalpy values from two individual binding steps.

titrating guests into a solution of $\mathrm{CB}[8]$, while values of $1: 1: 1$ heteroternary complexes were calculated from the sum of energies resulting from two individual binding steps: complexation of guest 1 into $\mathrm{CB}[8]$, followed by complexation of guest 2 into the $\mathrm{CB}[8]$-guest 1 solution.

As shown in Figure 1, the overall binding enthalpies for the formation of homoternary $(2: 1$, blue circles) and heteroternary (1:1:1, red circles) complexes fall in the range between -50 and $-80 \mathrm{~kJ} / \mathrm{mol}$. These values are consistent with computational studies of ternary complexes where two guest molecules occupy the inner cavity of $\mathrm{CB}[8]$ and lead to a complete dehydration of the cavity with enthalpy changes of $-66 \mathrm{~kJ} /$ mol. ${ }^{12}$

Complexes with a binding stoichiometry of 1:1 (green data sets in Figure 1) can be readily divided into two groups according to their $\Delta H$ values. A large majority of $1: 1$ complexes exhibited enthalpy changes under $-40 \mathrm{~kJ} / \mathrm{mol}$, which sit in the range expected for the formation of a binary complex, where the $\mathrm{CB}[8]$ cavity is partially dehydrated by accommodating only one guest. Although large variations are observed on account of different desolvation energies of guest molecules, ${ }^{28}$ a clear gap in enthalpy values of ca. $10 \mathrm{~kJ} / \mathrm{mol}$ is visible between binary and ternary complexes. Surprisingly, some complexes with a reported 1:1 stoichiometry exhibited larger enthalpy changes, ranging from -50 to $-80 \mathrm{~kJ} / \mathrm{mol}$, resembling values obtained for ternary complexes (Figure 1). These unique cases stem from three previous publications. The first was a study of $\mathrm{CB}[8]$ binding with diarylviologen derivatives (diamonds in Figure 1) published by Scherman et al. ${ }^{15}$ in 2012, while the second, describing $\mathrm{CB}[8]$ binding with several sequencespecific peptides (triangles in Figure 1), was reported by Urbach et al. ${ }^{16}$ in 2015. All of these complexes were regarded as binary in these two papers. Only in the third work (the squared-crossed marks in Figure 1), published in early 2016 by
Scherman et al., ${ }^{17}$ was a 2:2 quaternary complex described consisting of two azobenzene derivatives with two $\mathrm{CB}[8]$ macrocycles. Herein, we fully investigate the binding behavior of diarylviologens, exploring whether they exist as 2:2 quaternary or $1: 1$ binary complexes with $\mathrm{CB}[8]$.

\section{RESULTS AND DISCUSSION}

Diarylviologens with Electron-Donating Groups. Viologens, on account of their suitable size and electron-poor dicationic nature, are typically used as auxiliary first guests for $\mathrm{CB}[8]$, enabling complexation of a second guest, ${ }^{4}$ especially electron-rich aromatic species. ${ }^{5}$ The resulting donor-acceptor heteroternary complex was demonstrated to be an ideal model for studying charge-transfer (CT) phenomena. ${ }^{15}$ The energy level of $\mathrm{CB}[8]$-assisted CT pairings can be tuned by employing diarylviologens, which possess aryl moieties with substituents in the para position ranging from electron-donating groups (EDGs) to electron-withdrawing groups (EWGs). For a range of symmetric diarylviologens, abnormal enthalpy changes were observed $^{15}$ only from those with electron-donating groups (VEDGs), that is, $\mathrm{VH}, \mathrm{VMe}, \mathrm{VOMe}$, and $\mathrm{VNH}_{2}$, as shown in Figure 2a. This observation was confirmed by synthesizing

(a)
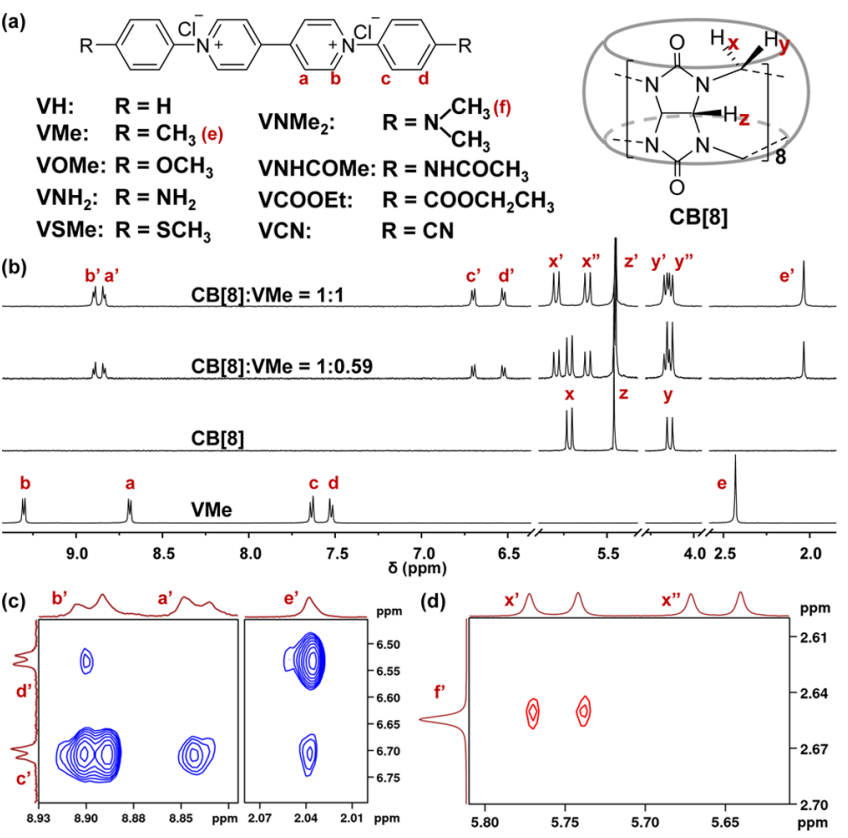

Figure 2. NMR studies for VEDG-CB[8] complexation. (a) Molecular structure of diarylviologen derivatives and $\mathrm{CB}[8]$. (b) Titration of VMe into $\mathrm{CB}[8]$ monitored by ${ }^{1} \mathrm{H}$ NMR, showing upfield shifts of $\mathrm{H}_{\mathrm{b}}-\mathrm{H}_{\mathrm{e}}$, a downfield shift of $\mathrm{H}_{\mathrm{a}}$, and splitting of $\mathrm{CB}[8]$ protons $\mathrm{H}_{\mathrm{x}}$ and $\mathrm{H}_{\mathrm{y}}$ (c) NOESY NMR of VMe-CB[8] showing additional off-diagonal correlations: $\mathrm{H}_{\mathrm{b}}^{\prime}-\mathrm{H}_{\mathrm{d}}^{\prime}, \mathrm{H}_{\mathrm{a}}^{\prime}-\mathrm{H}_{c}^{\prime}$, and $\mathrm{H}_{\mathrm{e}}^{\prime}-\mathrm{H}_{\mathrm{c}}^{\prime}$. (d) ROESY NMR of $\mathrm{VNMe}_{2}-\mathrm{CB}[8]$, showing correlations between methyl protons $\mathrm{H}_{\mathrm{f}}^{\prime}$ and $\mathrm{H}_{\mathrm{x}}^{\prime}$ but no correlations between $\mathrm{H}_{\mathrm{f}}^{\prime}$ and $\mathrm{H}_{\mathrm{x}}^{\prime \prime}$.

three additional VEDG derivatives, i.e., VSMe, $\mathrm{VNMe}_{2}$, and VNHCOMe (Figure 2a). ITC data of all VEDG derivatives were measured in buffered conditions (Figures S1-S7, Table $\mathrm{S} 1)$, resulting in enthalpy changes between -60 and $-80 \mathrm{~kJ} /$ mol (diamonds in Figure 1).

Conversely, diarylviologens bearing electron-withdrawing groups (VEWGs) exhibited 1:1 binding stoichiometry with $\mathrm{CB}[8]$ and an enthalpy change similar to that of a binary complex, which could readily uptake a second guest, forming a 
1:1:1 ternary complex. However, the complexes consisting of $\mathrm{CB}[8]$ and VEDG were unable to bind a second guest, displaying negligible exothermic heat in ITC as well as weak signals arising from ternary complex formation in electrospray ionization mass spectrometry; additionally, no change in fluorescence of the VMe-CB[8] complex was observed in the presence of a second guest. ${ }^{15}$ Most importantly, in the absence of an electron-rich second guest, $\mathrm{VNH}_{2}$ exhibited a significant absorption redshift of $100 \mathrm{~nm}$ upon $\mathrm{CB}[8]$ complexation. ${ }^{15}$ All of the above observations prompted us to revisit the binding manner between VEDG derivatives and $\mathrm{CB}[8]$.

2:2 Quaternary Complexes Deduced from ${ }^{1} \mathrm{H}$ NMR. NMR is the ideal technique to determine the relative position of guest molecules within CB complexes in solutions. Protons residing within the $\mathrm{CB}$ cavity typically exhibit a $1 \mathrm{ppm}$ upfield shift relative to unbound species, while protons proximate to the $\mathrm{CB}$ portal but not inside the cavity typically exhibit downfield shifts. $^{29}$ The titration of VMe into $\mathrm{CB}[8]$ was monitored by ${ }^{1} \mathrm{H}$ NMR as shown in Figure $2 \mathrm{~b}$. When forming the VMe-CB[8] complex, phenyl protons $\left(\mathrm{H}_{c}\right.$ and $\left.\mathrm{H}_{d}\right)$ exhibited a considerable upfield shift (ca. $1 \mathrm{ppm}, \mathrm{H}_{\mathrm{c}}^{\prime}$ and $\mathrm{H}_{\mathrm{d}}^{\prime}$ ), suggesting complete inclusion of the phenyl moiety inside the $\mathrm{CB}[8]$ cavity. One of the viologen protons $\left(\mathrm{H}_{\mathrm{b}}\right)$ as well as the methyl group protons $\left(\mathrm{H}_{\mathrm{e}}\right)$ also exhibited upfield shifts of ca. $0.4 \mathrm{ppm}\left(\mathrm{H}_{\mathrm{b}}^{\prime}\right.$ and $\left.\mathrm{H}_{\mathrm{e}}^{\prime}\right)$, suggesting an inclusion near the $\mathrm{CB}[8]$ portal or average signals arising from a partial inclusion and partial exclusion by the cavity. The other viologen proton $\left(\mathrm{H}_{\mathrm{a}}\right)$ showed a slight downfield shift of ca. $0.15 \mathrm{ppm}\left(\mathrm{H}_{\mathrm{a}}^{\prime}\right)$, suggesting a location near the portal yet outside the $\mathrm{CB}$ cavity. All of these shifts indicated that $\mathrm{CB}[8]$ bound with the tolyl moieties rather than the viologen in the middle of the VMe derivative. Similar patterns have been previously observed when two $\mathrm{CB}[7]$ hosts complexed both tolyl groups of a single VMe molecule forming a $2: 1$ complex. ${ }^{30}$ However, in the present case of $\mathrm{VMe}-\mathrm{CB}[8]$, the binding stoichiometry is clearly $1: 1$ rather than $2: 1$, confirmed through the integration of ${ }^{1} \mathrm{H}$ NMR as well as ITC (Figure S2). Moreover, the VMe-CB[8] complex exhibited slow dynamics (on the NMR time scale) compared to the VMe-CB $[7]_{2}$ complex, ${ }^{30}$ without any signal broadening observed during titration of the $\mathrm{VMe}$ into a solution of $\mathrm{CB}[8]$ (Figure 2b).

Interestingly, ${ }^{1} \mathrm{H}$ NMR spectra of all VEDG-CB [8] complexes (Figure 2b, Figures S9-S15) showed a significant splitting of $\mathrm{CB}[8]$ protons into sets of two equivalent peaks, particularly the $\mathrm{CB}[8]$ proton $\left(\mathrm{H}_{\mathrm{x}}\right)$ situated toward the interior of the cavity. To the best of our knowledge, such signal splitting has also been observed in the complexation of $\mathrm{CB}[6]$ with isobutylammonium, ${ }^{29} \mathrm{VMe}-\mathrm{CB}[7]_{2},{ }^{30} \mathrm{CB}[8]$ with two photocross-linked diaminostilbene derivatives, ${ }^{31} \mathrm{CB}[8]$ with a single diaminomelamine, ${ }^{32} \mathrm{CB}[8]$ with a U-shape bispyridinium derivatives, ${ }^{33} \mathrm{CB}[8]$ with a sequence-specific peptide, ${ }^{16}$ and two $\mathrm{CB}[8]$ with two azobenzene derivatives. ${ }^{17}$ The limited cases above have two things in common: (i) one CB portal is always exposed to more positive charge than the other, creating an asymmetric environment; (ii) importantly, the resulting complex exhibits slow dynamics in NMR.

Based on these observations, we propose a new $2: 2$ quaternary model for the $\mathrm{VMe}-\mathrm{CB}[8]$ complex as shown in Figure $3 \mathrm{a}$, in which two partially overlapping VMe derivatives are held in place by two $\mathrm{CB}[8]$ macrocycles around the aryl moieties. This binding model satisfies a 1:1 stoichiometry with two $\mathrm{CB}[8]$ hosts complexed with two VMe guests. Additionally, each $\mathrm{CB}[8]$ essentially accommodates two tolyl groups,

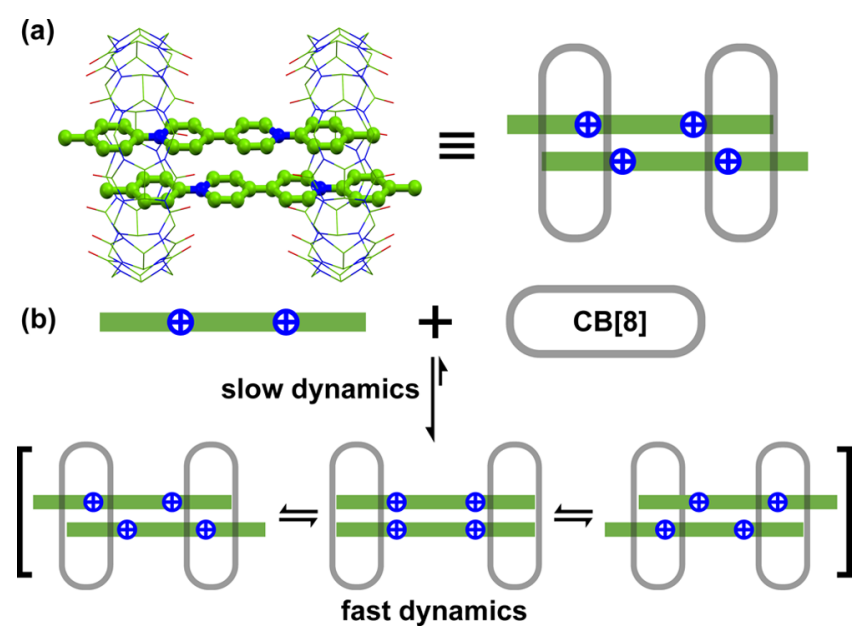

Figure 3. Schematic diagram of VEDG-CB $[8]$ complexation. (a) A molecular model proposed for 2:2 quaternary complexations: two partially overlapping VEDG molecules held in place by two $\mathrm{CB}[8]$ macrocycles. (b) Two dynamic processes involved in the 2:2 quaternary complexation: (i) slow inter-molecular association and dissociation and (ii) fast intra-conversion between different overlapping conformations.

which explains the abnormal enthalpy change (N.B. thermodynamic data in Figure 1 are reported on the basis of the complex containing one $\mathrm{CB}[8]$, i.e., $\left.[2 \mathrm{VMe}-2 \mathrm{CB}[8]]_{0.5}\right)$. This ultracooperative and tightly bound quaternary structure coincides with the sharp peaks and splitting patterns observed for the $\mathrm{CB}[8]$ proton signals during the NMR titration (Figure $2 \mathrm{~b}$ ), indicating slow exchange between bound and unbound $\mathrm{CB}[8]$ (Figure 3b). Moreover, on account of the head-to-head inclusion of two tolyl groups, one portal of each $\mathrm{CB}[8]$ is exposed to substantially higher density of positive charge than the other, in accordance with signal splitting of the $\mathrm{CB}[8]$ protons. We can further exclude formation of a supramolecular polymer; although a supramolecular polymer would also exhibit a 1:1 stoichiometry and large enthalpy change, its head-to-tail inclusion with symmetric portal environment cannot explain the observed NMR signal splitting. All other VEDG derivatives exhibit similar ${ }^{1} \mathrm{H}$ NMR patterns (Figures $59-S 15$ ) suggesting 2:2 quaternary complexation, with slight differences as expected by size and shape of substituents.

Evidence of Quaternary Complexation from 2D NMR. NOESY and ROESY 2D NMR were employed to further confirm our quaternary binding model and to investigate how the two VEDG molecules stack within the complex. In NOESY and ROSEY spectra, protons that are in close proximity to each other in space would exhibit off-diagonal correlations. The spectrum of unbound VMe molecules has positive off-diagonal correlations (red signals in Figure S18), whereas correlations in the spectrum of $\mathrm{VMe}-\mathrm{CB}[8]$ solutions are negative (blue signals in Figure 2c; full spectrum in Figure S19), which suggests a slow rotational exchange on account of the formation of a large structure.

Additional proton correlations, $\mathrm{H}_{\mathrm{b}}^{\prime}-\mathrm{H}_{\mathrm{d}}^{\prime}, \mathrm{H}_{\mathrm{a}}^{\prime}-\mathrm{H}_{\mathrm{c}}^{\prime}$, and $\mathrm{H}_{\mathrm{e}}^{\prime}-\mathrm{H}_{\mathrm{c}}^{\prime}$ were observed between viologen and tolyl moieties (Figure 2c), compared to the control NOESY spectrum of $\mathrm{VMe}$ without $\mathrm{CB}[8]$ where only a single correlation corresponding to $\mathrm{H}_{b}-\mathrm{H}_{c}$ was found (Figures S18 and S19). These inter-molecular correlations suggested that the tolyl group of one VMe in the complex should indeed be close to the viologen moiety from the other VMe molecule, and the complexed VMe molecules 
likely stack with each other in a parallel and partially overlapped manner, as shown in Figure 3a, rather than directly on top of each other.

In a partially overlapping structure, the two tolyl and pyridiumyl groups in the same VMe molecule should not be equivalent. However, the observation of only one set of signals for each proton in Figure $2 \mathrm{~b}$, suggest the protons are equivalent, which can only occur when the two VEDG guests undergo high frequency oscillations within the 2:2 quaternary complex. This intra-conversion between different overlapping conformations (Figure $3 \mathrm{~b}$ ) is too fast to be distinguished under the temporal resolution of NMR, resulting in one set of averaged signals.

From above studies we conclude that two dynamic processes are involved in the 2:2 quaternary complexation (Figure $3 \mathrm{~b}$ ): (i) slow inter-molecular association and dissociation, providing a stable complex or a strong linkage; (ii) fast intra-conversion between different overlapping conformations, displaying an oscillated figure.

Head-to-head inclusion was also verified by the ROESY $\mathrm{NMR}$ spectrum of the $\mathrm{VNMe}_{2}-\mathrm{CB}[8]$ complex, as shown in Figure 2d (Figure S23 for full spectrum), where the $\mathrm{H}_{\mathrm{f}}$ proton of the methyl group in $\mathrm{VNMe}_{2}$ only correlated with one of the two splitting $\mathrm{CB}[8]$ protons $\left(\mathrm{H}_{\mathrm{x}}\right)$, which means all the methyl groups are located near the same portal of one $\mathrm{CB}[8]$.

Structural Information from Ion Mobility Mass Spectrometry. In aqueous solution, complete desolvation of the $\mathrm{CB}[8]$ cavity makes $2: 2$ quaternary complexes the most energetically favorable species. In the gas phase, however, 1:1 binary complexes are the most stable species, as without desolvation the complexation is only dominated by ion-dipole interactions, and is no longer correlated with the affinity in solution. ${ }^{34,35}$ This is why the dominant species observed in the mass spectra of VEDG-CB[8] systems is the $1: 1$ binary complex, which likely led to the inappropriate binding model in previous studies. ${ }^{15,36}$

In fact, signals from 2:2 quaternary species were readily observed in all VEDG-CB[8] solutions (Figures S25-S32) with the help of ion mobility mass spectrometry (IM-MS). IMMS is a technique that can identify molecules in the gas phase not only according to their mass and charge, but also according to their size and shape. In IM-MS, ionized species will migrate through a chamber containing a neutral gas (e.g., helium or nitrogen); large ions will have more collisions with the neutral gas, thus taking more time (drift time) to traverse the chamber in comparison with smaller ions. Therefore, the drift time from IM-MS supplies an additional dimension to separate and distinguish ions with high resolution (Figure S24). ${ }^{37,38}$

On account of the dissociation (to 1:1 binary complex) in the gas phase, 2:2 quaternary species exhibited weak signals and typically only one charge state $\left(3^{+}\right)$. The exception was VNHCOMe, which exhibited a higher intensity of 2:2 species than other VEDG, enabling the observation of more charge states including $3^{+}, 4^{+}$, and $5^{+}$(Figures S24 and S25). This is likely because the larger substituent group of VNHCOMe extends the lifetime of 2:2 quaternary complex and suppresses dissociation during the transition from aqueous solution to the gas phase.

In addition to mass and charge, IM-MS also supplies structural information for identified molecules. ${ }^{37,38}$ For all VEDG and VCOOEt studied here, upon injection of their aqueous solutions containing a slight excess of $\mathrm{CB}[8]$, one could observe $\mathrm{m} / \mathrm{z}$ signals corresponding to diarylviologens
(V), single $\mathrm{CB}[8](1 \mathrm{CB}[8]), 1: 1$ binary complex $(1 \mathrm{~V}-1 \mathrm{CB}[8])$, dimer of $\mathrm{CB}[8](2 \mathrm{CB}[8]), 1: 2$ complex (1V-2CB[8]), and 2:2 quaternary complex $(2 \mathrm{~V}-2 \mathrm{CB}[8])$ (Figures S24-S32). Size and shape information on each ion in the gas phase $\left(\mathrm{N}_{2}\right)$ was represented by the rotationally averaged collision cross-section (CCS), derived from drift time measured from IM-MS. ${ }^{39,40}$ The CCS of ionic species that contained $\mathrm{CB}[8]$ was plotted against the CCS of diarylviologen dications $\left(\mathrm{V}^{2+}\right)$, as shown in Figure 4. As ionic species from $\mathrm{CB}[8]$ are present in all

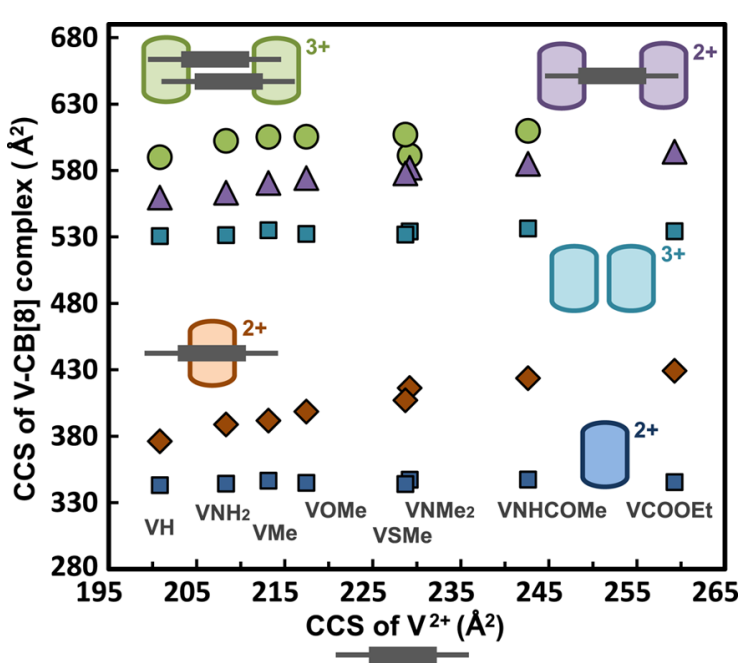

Figure 4. Collision cross-section (CCS) of species that involved $\mathrm{CB}[8]$ against the $\mathrm{CCS}$ of $\mathrm{V}^{2+}$. Species included $[1 \mathrm{CB}[8]]^{2+}$ (blue squares), $[1 \mathrm{~V}-1 \mathrm{CB}[8]]^{2+}$ (brown diamonds), $[2 \mathrm{CB}[8]]^{3+}$ (cyan squares), $[1 \mathrm{~V}-2 \mathrm{CB}[8]]^{2+}$ (purple triangles), and $[2 \mathrm{~V}-2 \mathrm{CB}[8]]^{3+}$ (green circles). CCS was determined by ion mobility mass spectrometry using $\mathrm{N}_{2}$ as collision gas and polyalanine as calibration ion. $[2 \mathrm{~V}-2 \mathrm{CB}[8]]^{3+}$ for VCOOEt was not observed.

samples, the validity of $\mathrm{CCS}$ values are certified by $[1 \mathrm{CB}[8]]^{2+}$ (blue squares) and $[2 \mathrm{CB}[8]]^{3+}$ (cyan squares), whose CCS are uniform for different samples at $345 \pm 2$ and $533 \pm 2 \AA^{2}$, respectively.

In a $1: 1 \mathrm{~V}-\mathrm{CB}[8]$ complex, the $\mathrm{CB}[8]$ macrocycle tends to bind with dicationic viologen moiety, while for aryl substituents, which are outside the $\mathrm{CB}[8]$ cavities, their size will be reflected in CCS values through the gas collision. Figure 4 demonstrates that the CCS of $[1 \mathrm{~V}-1 \mathrm{CB}[8]]^{2+}$ (brown diamonds) increases with the CCS of $\mathrm{V}^{2+}$ (horizontal axis). Conversely, the CCS values of $[2 \mathrm{~V}-2 \mathrm{CB}[8]]^{3+}$ (green circles) for all VEDG derivatives are not susceptible to the size of their substituents, suggesting that in a 2:2 quaternary complex $\mathrm{CB}$ [8] binds the aryl substituents and screens them from gas collision, therefore, exhibiting similar CCS. This is consistent with the observation from ${ }^{1} \mathrm{H}$ NMR (Figure $2 b$ ) for 2:2 quaternary complex that the phenyl moieties of VEDG are encapsulated inside $\mathrm{CB}[8]$ cavity.

CCS values can be calculated using helium as the collision gas through the projection approximation method. As shown in Figure S34, the CCS calculated for 2:2 quaternary models containing two head-to-head stacked VEDG molecules are around $570 \AA^{2}$, and are similar to the value for $1 \mathrm{~V}-2 \mathrm{CB}[8]$ complex (N.B. CCS in He gas is slightly smaller than that in $\mathrm{N}_{2}$ gas). Even when taking the dynamic intra-conversion into consideration (Figure 3b), two VEDG molecules with a different extent of overlap only lead to a small variation of CCS values. However, a 2:2 complex containing head-to-tail 


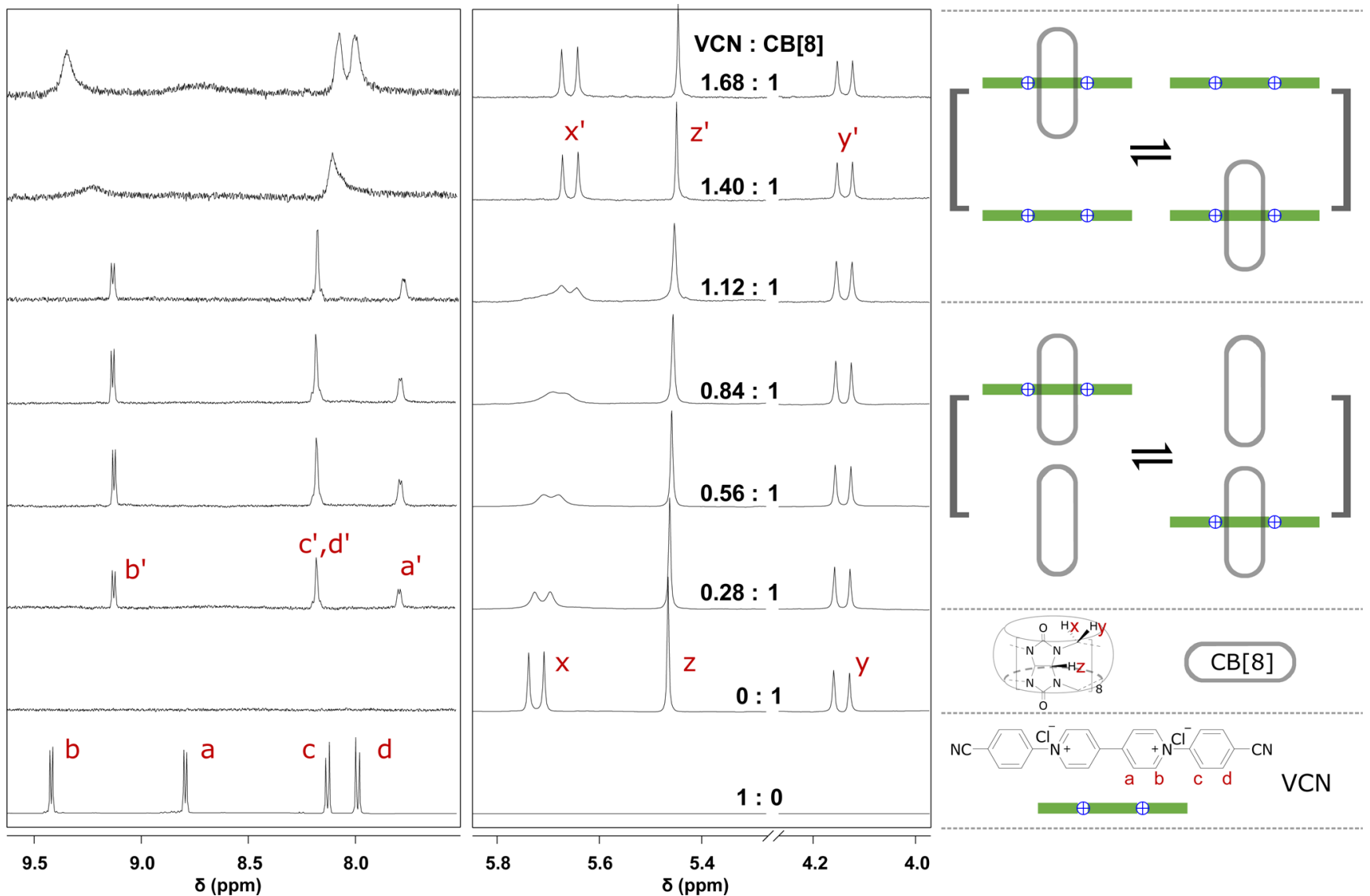

Figure 5. NMR studies for VCN-CB[8] complexation. Titration of VCN into $\mathrm{CB}[8]$ monitored by ${ }^{1} \mathrm{H}$ NMR exhibits upfield shifts of $\mathrm{H}_{\mathrm{a}}$ and $\mathrm{H}_{\mathrm{b}}$, downfield shifts of $\mathrm{H}_{\mathrm{c}}$ and $\mathrm{H}_{\mathrm{d}}$, and no splitting of $\mathrm{CB}[8]$ protons $\left(\mathrm{H}_{\mathrm{x}}\right.$ and $\left.\mathrm{H}_{\mathrm{y}}\right)$, indicating a 1:1 binary complexation. During the titration, the signal of $\mathrm{CB}[8]$ proton $\mathrm{H}_{\mathrm{x}}$ was broad at first, on account of the fast exchange between bound and unbound $\mathrm{CB}[8]$, and then became sharp as there was almost no unbound $\mathrm{CB}[8]$ in present of an excess of $\mathrm{VCN}$.

stacking of two VEDG molecules results in a calculated $\mathrm{CCS}(\mathrm{He})$ larger than $700 \AA^{2}$. The experimental $\mathrm{CCS}\left(\mathrm{N}_{2}\right)$ values of $[2 \mathrm{~V}-2 \mathrm{CB}[8]]^{3+}$ (green circles, Figure 4$)$ are around $600 \AA^{2}$, and slightly larger than the CCS of $[1 \mathrm{~V}-2 \mathrm{CB}[8]]^{2+}$ (purple triangles). These results, therefore, confirm the headto-head stacking of two VEDG molecules in a 2:2 quaternary complex.

Why EWGs Do Not Favor Formation of Quaternary Complexes. Among all diarylviologens prepared, including VEDG, VEWG, and VX (X = F, Cl, Br, I), ${ }^{15}$ only VEDG derivatives were found to form 2:2 quaternary complexes with $\mathrm{CB}[8]$. In the IM-MS, a $m / z$ signal corresponding to the $2 \mathrm{~V}$ $2 \mathrm{CB}[8]$ species was not observed for VCOOEt, even though it possessed a larger substituent group than VNHCOMe. ${ }^{1} \mathrm{H}$ NMR of VCN (Figure 5) and VCOOEt (Figure S16) with $\mathrm{CB}[8]$, both exhibited a large upfield shift of the viologen protons and a slight downfield shift of the phenyl protons typical for a $1: 1$ binary complex in which the $\mathrm{CB}[8]$ is bound on the viologen moiety. Moreover, the resulting complex exhibited a highly dynamic binding in NMR. In the presence of excess $\mathrm{CB}[8]$, as shown in Figure 5, the signal of $\mathrm{CB}[8]$ proton $\mathrm{H}_{\mathrm{x}}$ was broad on account of the fast exchange between bound and unbound $\mathrm{CB}[8]$, and exhibited a chemical shift averaged from bound and unbound species. When excess VCN was added, the $\mathrm{H}_{\mathrm{x}}$ signal became sharp, and instead signals corresponding to $\mathrm{VCN}$ protons became broad. The results discussed above as well as the previously reported binding enthalpy ${ }^{15}$ all verify the tendency of VEWGs to form 1:1 binary complexes.

In order to align two viologens moieties close to each other, repulsion between positive charges must be overcome, for instance by reduction of viologen to its radical cation form. ${ }^{41}$ Repulsion may also be mitigated by directly connecting viologen units to electron-rich moieties and dispersing the charges into a large conjugated structure. Therefore, the 2:2 quaternary motif is only favored by diarylviologens carrying electron-donating group. Viologens with electron-withdrawing groups cannot readily disperse their positive charges, but rather concentrate them locally. This is fully consistent with the observation that VEWG derivatives are not stable in the presence of moisture and $\mathrm{O}_{2}$.

Specific Properties Arising from a 2:2 Quaternary Structure. The observation and deeper understanding of the formation of 2:2 quaternary complexes is not merely an academic exercise or a correction of previously published misinterpretation of data; but also carries fundamental implications for the design of functional materials. First, the twisted orientation between the two aryl substituents and the central viologen moiety as well as the twisted orientation between the pyridium groups of the viologen itself will be substantially reduced (flattened) in a confined space (i.e., the CB cavity), rendering an extended highly conjugated structure. $^{30}$ Second, the two VEDG molecules can readily interact electronically forming a charge-transfer pair, exhibiting 
a much larger overlap of molecular orbitals than a $1: 1: 1 \mathrm{CB}[8]$ mediated heteroternary complex.

On account of the extended conjugated structure, a significant bathochromic shift was observed for all VEDG$\mathrm{CB}[8]$ complexes when compared to the absorption of VEDG without $\mathrm{CB}[8]$, as shown in Figures S35-S41, examplified by $\mathrm{VNMe}_{2}$ in Figure 6a. A dramatic change in solution color could
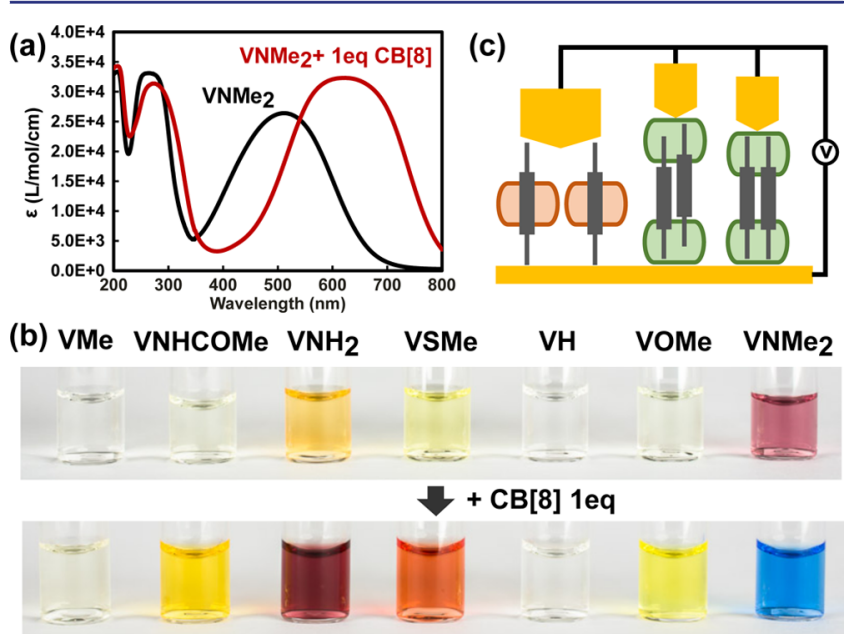

Figure 6. Photophysical properties of quaternary structures. (a) Absorption of $\mathrm{VNMe}_{2}$ in $\mathrm{H}_{2} \mathrm{O}(50 \mu \mathrm{M})$ before and after addition of 1 equiv of $\mathrm{CB}[8]$. (b) Color change observed in VEDG solutions before and after addition of 1 equiv of $\mathrm{CB}[8]$. (c) Previous 1:1 binding model (left) and new 2:2 quaternary models (middle and right) for the single-molecule conductance of VSMe-CB $[8]$ complex.

be observed, especially with heteroatoms such as $\mathrm{S}, \mathrm{N}$, or $\mathrm{O}$ directly attached to the phenyl group (Figure $6 \mathrm{~b}$ ). While absorption changes for $\mathrm{VNH}_{2}{ }^{15}$ and $\mathrm{VSMe}^{36}$ were previously reported upon $\mathrm{CB}[8]$ complexation, no explanation was provided, as an improper binary binding model was employed in both reports.

We hypothesize that the rigid and highly conjugated structure in the quaternary complex will favor transport of electrons and act as an excellent molecular conductor. Recently, Zhang et al. ${ }^{36}$ reported that the single molecule conductance of viologen derivatives could be increased by forming a complex with $\mathrm{CB}[8]$, where VSMe was employed. In their study, the conductance of the molecular junction from VSMe in water was $1.57 \mathrm{nS}$ measured using a non-contact STM technique. In a solution containing equal amounts of VSMe and $\mathrm{CB}[8]$, upon approach of the STM tip to the substrate, an initial peak of 4.2 $\mathrm{nS}$ was observed, with a second peak of $8.0 \mathrm{nS}$ found by further decreasing the tip-substrate distance. Although they attributed these two peaks to stochastic formation of "double junctions", based on the binary binding model shown in Figure 6c (left), Zhang et al. ${ }^{36}$ could not explain why double junctions were frequently observed with VSMe, but rarely seen in other viologens derivatives investigated.

The 2:2 quaternary complexation model we propose here readily explains the striking increase of conductance as well as the observation of an additional conducting signal. The extended, highly conjugated structure and large overlap of molecular orbitals favors transport of electrons, responsible for the remarkable molecular conductance observed in the VSMe$\mathrm{CB}[8]$ system. The two VEDG molecules are partially overlapping with each other, as depicted in Figure 6c (middle), when the tip moves toward the substrate, it first comes into contact with one VEDG. As it continues to move toward the substrate, the tip is able to simultaneously contact two VEDG molecules or alternatively force the two VEDG molecules to completely overlap in the quaternary structure as shown in Figure $6 c$ (right). In both cases, the result is a doubling of the cross-sectional area of this "supramolecular" conductor, similar to a parallel circuit, and therefore doubles the conductance from 4.2 to $8.0 \mathrm{nS}$. As performed by Zhang et al., ${ }^{36}$ the association of either alkyl or aromatic viologens with $\mathrm{CB}[8]$ can generally enhance conductance at the single molecule level as the $\mathrm{CB}[8]$ cavity may suppress the twist of viologen moieties. However, only 2:2 quaternary complexes can exhibit a remarkable increase in conductance, almost twice the value of alkyl viologen- $\mathrm{CB}[8]$ complexes, which suggests the potential application of 2:2 complex as optoelectronic materials.

\section{CONCLUSION}

Through revisiting previously reported binding enthalpies for $\mathrm{CB}[8]$-mediated host-guest complexation, we found that among the complexes with 1:1 stoichiometry, diarylviologens containing electron-donating substituents exhibited abnormally large binding enthalpies, similar in value to the formation of ternary complexes rather than binary complexes. We demonstrated that these compounds indeed form unnoticed 2:2 quaternary complexes by threading two highly conjugated viologens into two $\mathrm{CB}[8]$ cavities. EDG substituents were capable of reducing the coulomb repulsion of the parallel stacked viologen moieties by dispersing the positive charges. Thus, the stacking of two diarylviologens in a highly conjugated conformation affords 2:2 quaternary complexes with unique photophysical properties, and provides substantial new understanding and insight.

It is noteworthy that the observation of abnormally large binding enthalpies as well as the splitting of $\mathrm{CB}[8]$ proton signals alone may not be sufficient for verifying 2:2 quaternary complexes, especially when a guest molecule possesses a flexible backbone. For instance, 1:1 complexes with large binding enthalpies (triangles in Figure 1) were interpreted by folding sequence-specific peptides into a compact conformation that could occupy the whole $\mathrm{CB}[8]$ cavity; $^{16}$ splitting of $\mathrm{CB}[8]$ proton signals observed in a 1:1 bispyridinium-CB $[8]$ complex was alternatively explained as a U-shaped guest clamped by one $\mathrm{CB}[8]$ macrocycle. ${ }^{33}$ However, as neither of these reports has convincingly ruled out the formation of $2: 2$ quaternary complexes, it is worth reinvestigating the binding behavior of these guests, exploring whether they exist as 2:2 quaternary or 1:1 compacted complexes with $\mathrm{CB}[8]$.

Although this is not the first observation that a complex of 1:1 binding stoichiometry contains more than one host and one guest (e.g., a 5:5 molecular necklace ${ }^{42}$ or an $n: n$ supramolecular polymer $\left.{ }^{18}\right)$, the $2: 2$ complexes observed in this work exhibit quaternary structures with noticeable stability in solution. This unique feature on account of slow dynamics along with its strong binding affinity will ensure numerous applications involving design and fabrication of supramolecular polymers, viscoelastic networks, and other engineered nanostructures. It may provide an ideal model system for the study and critical understanding of electron transport on a single molecule level, with development of functional optoelectronic materials exhibiting desirable and tunable properties. 


\section{ASSOCIATED CONTENT}

\section{S Supporting Information}

The Supporting Information is available free of charge on the ACS Publications website at DOI: 10.1021/jacs.6b13074.

Materials and methods; synthesis and characterization of diarylviologens; isothermal titration thermograms of VEDG; thermodynamic data of $\mathrm{CB}[8]$-mediated complexes of $1: 1,1: 1: 1$, and $2: 1$ stoichiometry; ${ }^{1} \mathrm{H}$ NMR titration of diarylviologens into $\mathrm{CB}[8]$; $2 \mathrm{D} \mathrm{NMR}$ of $\mathrm{VMe}$ and $\mathrm{VNMe}_{2}$ before and after addition of $\mathrm{CB}[8]$; mass spectra of $\mathrm{V}-\mathrm{CB}[8]$ complexes; collision cross-section of $\mathrm{V}-\mathrm{CB}[8]$ complexes; UV/vis spectra of VEDG before and after addition of $\mathrm{CB}[8]$; and ${ }^{1} \mathrm{H}$ and ${ }^{13} \mathrm{C}$ NMR spectra of $\mathrm{VNMe}_{2}$ and $\mathrm{VNHCOMe}$, including Figures S1-S45 and Tables S1-S5 (PDF)

\section{AUTHOR INFORMATION}

\section{Corresponding Author}

*oas23@cam.ac.uk

ORCID ${ }^{\circ}$

Oren A. Scherman: 0000-0001-8032-7166

Author Contributions

${ }^{\S}$ G.W. and M.O. contributed equally to this work.

Notes

The authors declare no competing financial interest.

\section{ACKNOWLEDGMENTS}

The authors thank the Leverhulme Trust (project: 'Natural material innovation for sustainable living'), the Marie Curie FP7 SASSYPOL ITN (607602) programme, and EPSRC (EP/ L504920/1) for funding. The authors thank Dr. Peter Grice and Mr. Duncan Howe for their help on NMR analysis, and Dr. Seán Ryan and Dr. David Clarke for their kind suggestion.

\section{REFERENCES}

(1) Day, A.; Arnold, A. P.; Blanch, R. J.; Snushall, B. J. Org. Chem. 2001, 66, 8094-8100.

(2) Lee, J. W.; Samal, S.; Selvapalam, N.; Kim, H.-J.; Kim, K. Acc. Chem. Res. 2003, 36, 621-630.

(3) Lagona, J.; Mukhopadhyay, P.; Chakrabarti, S.; Isaacs, L. Angew. Chem., Int. Ed. 2005, 44, 4844-4870.

(4) Kim, H.-J.; Heo, J.; Jeon, W. S.; Lee, E.; Kim, J.; Sakamoto, S.; Yamaguchi, K.; Kim, K. Angew. Chem., Int. Ed. 2001, 40, 1526-1529.

(5) Rauwald, U.; Biedermann, F.; Deroo, S.; Robinson, C. V.; Scherman, O. A. J. Phys. Chem. B 2010, 114, 8606-8615.

(6) Heitmann, L. M.; Taylor, A. B.; Hart, P. J.; Urbach, A. R. J. Am. Chem. Soc. 2006, 128, 12574-12581.

(7) Barrow, S. J.; Kasera, S.; Rowland, M. J.; del Barrio, J.; Scherman, O. A. Chem. Rev. 2015, 115, 12320-12406.

(8) Biedermann, F.; Nau, W. M. Angew. Chem., Int. Ed. 2014, 53, 5694-5699.

(9) Assaf, K. I.; Nau, W. M. Chem. Soc. Rev. 2015, 44, 394-418.

(10) Masson, E.; Ling, X.; Joseph, R.; Kyeremeh-Mensah, L.; Lu, X. RSC Adv. 2012, 2, 1213-1247.

(11) Gürbüz, S.; Idris, M.; Tuncel, D. Org. Biomol. Chem. 2015, 13, 330-347.

(12) Biedermann, F.; Uzunova, V. D.; Scherman, O. A.; Nau, W. M.; De Simone, A. J. Am. Chem. Soc. 2012, 134, 15318-15323.

(13) Biedermann, F.; Vendruscolo, M.; Scherman, O. A.; De Simone, A.; Nau, W. M. J. Am. Chem. Soc. 2013, 135, 14879-14888.

(14) Biedermann, F.; Nau, W. M.; Schneider, H.-J. Angew. Chem., Int. Ed. 2014, 53, 11158-11171.

(15) Biedermann, F.; Scherman, O. A. J. Phys. Chem. B 2012, 116, $2842-2849$.
(16) Smith, L. C.; Leach, D. G.; Blaylock, B. E.; Ali, O. A.; Urbach, A. R. J. Am. Chem. Soc. 2015, 137, 3663-3669.

(17) del Barrio, J.; Ryan, S. T.; Jambrina, P. G.; Rosta, E.; Scherman, O. A. J. Am. Chem. Soc. 2016, 138, 5745-5748.

(18) Liu, Y.; Yu, Y.; Gao, J.; Wang, Z.; Zhang, X. Angew. Chem., Int. Ed. 2010, 49, 6576-6579.

(19) Biedermann, F.; Rauwald, U.; Cziferszky, M.; Williams, K. A.; Gann, L. D.; Guo, B. Y.; Urbach, A. R.; Bielawski, C. W.; Scherman, O. A. Chem. - Eur. J. 2010, 16, 13716-13722.

(20) Bush, M. E.; Bouley, N. D.; Urbach, A. R. J. Am. Chem. Soc. 2005, 127, 14511-14517.

(21) Huang, Z.; Yang, L.; Liu, Y.; Wang, Z.; Scherman, O. A.; Zhang, X. Angew. Chem., Int. Ed. 2014, 53, 5351-5355.

(22) Liu, Y.; Fang, R.; Tan, X.; Wang, Z.; Zhang, X. Chem. - Eur. J. 2012, 18, 15650-15654

(23) Ko, Y. H.; Kim, Y.; Kim, H.; Kim, K. Chem. - Asian J. 2011, 6, 652-657.

(24) Baek, K.; Kim, Y.; Kim, H.; Yoon, M.; Hwang, I.; Ko, Y. H.; Kim, K. Chem. Commun. 2010, 46, 4091-4093.

(25) Zhang, Y.; Zhou, T.-Y.; Zhang, K.-D.; Dai, J.-L.; Zhu, Y.-Y.; Zhao, X. Chem. - Asian J. 2014, 9, 1530-1534.

(26) Biedermann, F.; Ross, I.; Scherman, O. A. Polym. Chem. 2014, 5, $5375-5382$

(27) Huang, Z.; Qin, K.; Deng, G.; Wu, G.; Bai, Y.; Xu, J.-F.; Wang, Z.; Yu, Z.; Scherman, O. A.; Zhang, X. Langmuir 2016, 32, 1235212360 .

(28) Biedermann, F.; Schneider, H.-J. Chem. Rev. 2016, 116, 52165300.

(29) Mock, W. L.; Shih, N. Y. J. Org. Chem. 1986, 51, 4440-4446.

(30) Freitag, M.; Gundlach, L.; Piotrowiak, P.; Galoppini, E. J. Am. Chem. Soc. 2012, 134, 3358-3366.

(31) Jon, S. Y.; Ko, Y. H.; Park, S. H.; Kim, H.-J.; Kim, K. Chem. Commun. 2001, 1938-1939.

(32) Liu, S.; Ruspic, C.; Mukhopadhyay, P.; Chakrabarti, S.; Zavalij, P. Y.; Isaacs, L. J. Am. Chem. Soc. 2005, 127, 15959-15967.

(33) Zhang, Z.-J.; Zhang, Y.-M.; Liu, Y. J. Org. Chem. 2011, 76, $4682-4685$.

(34) Lee, S. J. C.; Lee, J. W.; Lee, H. H.; Seo, J.; Noh, D. H.; Ko, Y. H.; Kim, K.; Kim, H. I. J. Phys. Chem. B 2013, 117, 8855-8864.

(35) Lee, J. W.; Lee, H. H. L.; Ko, Y. H.; Kim, K.; Kim, H. I. J. Phys. Chem. B 2015, 119, 4628-4636.

(36) Zhang, W.; Gan, S.; Vezzoli, A.; Davidson, R. J.; Milan, D. C.; Luzyanin, K. V.; Higgins, S. J.; Nichols, R. J.; Beeby, A.; Low, P. J.; Li, B.; Niu, L. ACS Nano 2016, 10, 5212-5220.

(37) Ruotolo, B. T.; Benesch, J. L.; Sandercock, A. M.; Hyung, S.-J.; Robinson, C. V. Nat. Protoc. 2008, 3, 1139-1152.

(38) Hofmann, J.; Hahm, H.; Seeberger, P. H.; Pagel, K. Nature 2015, 526, 241-244.

(39) Campuzano, I.; Bush, M. F.; Robinson, C. V.; Beaumont, C.; Richardson, K.; Kim, H.; Kim, H. I. Anal. Chem. 2012, 84, 1026-1033.

(40) Paglia, G.; Williams, J. P.; Menikarachchi, L.; Thompson, J. W.; Tyldesley-Worster, R; Halldórsson, S.; Rolfsson, O.; Moseley, A.; Grant, D.; Langridge, J.; Palsson, B. O.; Astarita, G. Anal. Chem. 2014, 86, 3985-3993.

(41) Jeon, W. S.; Kim, H.-J.; Lee, C.; Kim, K. Chem. Commun. 2002, $1828-1829$.

(42) Ko, Y. H.; Kim, K.; Kang, J.-K.; Chun, H.; Lee, J. W.; Sakamoto, S.; Yamaguchi, K.; Fettinger, J. C.; Kim, K. J. Am. Chem. Soc. 2004, 126, 1932-1933. 